\title{
Assessment of the Lymph Node Status in Patients Undergoing Liver Resection for Intrahepatic Cholangiocarcinoma: the New Eighth Edition AJCC Staging System
}

\author{
Fabio Bagante ${ }^{1} \cdot$ Gaya Spolverato $^{1} \cdot$ Matthew Weiss $^{2} \cdot$ Sorin Alexandrescu ${ }^{3}$. \\ Hugo P. Marques ${ }^{4}$. Luca Aldrighetti ${ }^{5}$. Shishir K. Maithel ${ }^{6}$. Carlo Pulitano ${ }^{7}$. \\ Todd W. Bauer ${ }^{8}$ Feng Shen ${ }^{9}$ George A. Poultsides ${ }^{10}$ - Oliver Soubrane ${ }^{11}$ • \\ Guillaume Martel $^{12}$ • B. Groot Koerkamp ${ }^{13}$ - Alfredo Guglielmi ${ }^{1}$ - Endo Itaru $^{14}$. \\ Timothy M. Pawlik ${ }^{15}$
}

Received: 16 February 2017 / Accepted: 6 April 2017 /Published online: 19 April 2017

(C) 2017 The Society for Surgery of the Alimentary Tract

\begin{abstract}
Introduction The role of routine lymphadenectomy for intrahepatic cholangiocarcinoma (ICC) is still controversial. The AJCC eighth edition recommends a minimum of six harvested lymph nodes (HLNs) for adequate nodal staging. We sought to define outcome and risk of death among patients who were staged with $\geq 6$ HLNs versus $<6$ HLNs.

Materials and Methods Patients undergoing hepatectomy for ICC between 1990 and 2015 at 1 of the 14 major hepatobiliary centers were identified.

Results Among 1154 patients undergoing hepatectomy for ICC, 515 (44.6\%) had lymphadenectomy. On final pathology, 200 (17.3\%) patients had metastatic lymph node (MLN), while 315 (27.3\%) had negative lymph node (NLN). Among NLN patients, HLN was associated with 5-year OS $(p=0.098)$. While HLN did not impact 5-year OS among MLN patients $(p=0.71)$, the number of MLN was associated with 5-year OS $(p=0.02)$. Among the $317(27.5 \%)$ patients staged according the AJCC eighth edition staging system, $\mathrm{N} 1$ patients had a 3 -fold increased risk of death compared with N0 patients (hazard ratio 3.03; $p<0.001$ ).
\end{abstract}

Gaya Spolverato and Fabio Bagante contributed equally to this work.

Electronic supplementary material The online version of this article (doi:10.1007/s11605-017-3426-x) contains supplementary material, which is available to authorized users.

Timothy M. Pawlik

tim.pawlik@osumc.edu

1 Department of Surgery, University of Verona, Verona, Italy

2 Department of Surgery, Johns Hopkins Hospital, Baltimore, MD, USA

3 Department of Surgery, Fundeni Clinical Institute, Bucharest, Romania

4 Department of Surgery, Curry Cabral Hospital, Lisbon, Portugal

5 Department of Surgery, Ospedale San Raffaele, Milan, Italy

6 Department of Surgery, Emory University, Atlanta, GA, USA

7 Department of Surgery, Royal Prince Alfred Hospital, University of Sydney, Sydney, Australia

8 Department of Surgery, University of Virginia, Charlottesville, VA, USA

9 Department of Surgery, Eastern Hepatobiliary Surgery Hospital, Shanghai, China

10 Department of Surgery, Stanford University, Stanford, CA, USA

11 Department of Hepatobiliopancreatic Surgery and Liver Transplantation, AP-HP, Beaujon Hospital, Clichy, France

12 Division of General Surgery, Department of Surgery, University of Ottawa, Ottawa, ON, Canada

13 Department of Surgery, Erasmus University Medical Centre, Rotterdam, Netherlands

14 Gastroenterological Surgery Division, Yokohama City University School of Medicine, Yokohama, Japan

15 Department of Surgery, The Urban Meyer III and Shelley Meyer Chair in Cancer Research, The Ohio State University Wexner Medical Center, 395 W. 12th Ave., Suite 670, Columbus, OH 43210 , USA 
Conclusion Only one fourth of patients undergoing hepatectomy for ICC had adequate nodal staging according to the AJCC eighth edition. While the six HLN cutoff value impacted prognosis of N0 patients, the number of MLN rather than HLN was associated with long-term survival of N1 patients.

Keywords ICC $\cdot$ Surgery $\cdot$ Staging $\cdot$ Nodal status

\section{Introduction}

The American Joint Committee on Cancer (AJCC) staging manual is the most common means to stratify cancer patients with regard to prognosis. In the seventh edition AJCC staging manual, a staging system for ICC was introduced for the first time. The newly released eighth edition AJCC T categories have several modifications for ICC staging, including the addition of tumor size to lesion number and vascular invasion $\left[{ }^{1-7}\right]$. AJCC nodal $(\mathrm{N})$ staging has also been a topic of ongoing debate, as the role for routine lymphadenectomy for ICC has been controversial with no standard approach to assessing regional nodal information $[2,8,9]$. In one study using the Surveillance, Epidemiology, and End Results (SEER) cancer registry, Kim et al. reported on 749 patients who underwent surgical resection of ICC between 1988 and $2011\left[^{10}\right]$. In this study, Kim et al. assessed the prognostic performance of AJCC/UICC seventh N stage, lymph node ratio (LNR), and $\log$ odds (LODDS) among patients with ICC $\left[{ }^{10}\right]$. Interestingly, after curative intent resection of ICC, LODDS and LNR were better predictors of long-term prognosis versus seventh edition AJCC nodal staging. In particular, while LNR performed well among patients who had $>3$ LNs harvested and examined, LODDS was better at determining prognosis among patients with $\leq 3 \mathrm{LN}$ examined.

While nodal status appears to be an important factor in determining prognosis of patients with ICC $\left[{ }^{11,}{ }^{12}\right]$, routine lymphadenectomy is not always performed, especially in Western centers $\left[{ }^{13,14}\right]$. Data on pathological lymph node status are, therefore, often lacking. Importantly, guidelines of the European Association for the Study of the Liver (EASL) on the management of ICC recommend the removal of lymph nodes defined as suspicious according to preoperative imaging $\left[{ }^{15}\right]$. However, the correlation of radiological lymph node status assessment with pathological $\mathrm{N}$ status has yet to be determined. As such, the objective of the current study was to correlate the performance of radiological versus pathological assessment of lymph node status among patients with resectable ICC. Moreover, given that the newly released AJCC eighth edition recommends the recovery of at least six lymph nodes for complete pathologic staging, we sought to define the outcome of patients who were "adequately" ( $\geq 6$ nodes harvested) versus "inadequately" staged ( $<6$ nodes harvested) according to the eighth edition of the AJCC staging manual $\left[{ }^{16}\right]$.

\section{Materials and Methods}

\section{Patient Demographic and Clinical Data}

Patients undergoing liver surgery for histologically confirmed ICC between 1990 and 2015 at one of the following 14 major hepatobiliary centers were identified: Johns Hopkins Hospital, Baltimore, MD; Stanford University, Stanford, CA; University of Virginia, Charlottesville, VA; Emory University, Atlanta, GA; Fundeni Clinical Institute of Digestive Disease, Bucharest, Romania; Curry Cabral Hospital, Lisbon, Portugal; Ospedale San Raffaele, Milan, Italy; Royal Prince Alfred Hospital, University of Sydney, Sydney, Australia; Eastern Hepatobiliary Surgery Hospital, Shanghai, China; Beaujon Hospital, Clichy, France; University of Ottawa, Ottawa, Ontario, Canada; Erasmus University Medical Centre, Rotterdam, Netherlands; Yokohama City University School of Medicine, Yokohama, Japan; and University of Verona, School of Medicine, Verona, Italy. The Institutional Review Board of each institution approved the study. Only patients who underwent curative intent surgery were included, while patients with metastatic disease and patients who underwent an R2 resection were excluded.

Standard patient demographic and clinicopathologic data were collected including age, sex, American Society of Anesthesiologists (ASA) physical status classification, and presence of underlying liver disease, such as cirrhosis, chronic hepatitis B infection, and chronic hepatitis $\mathrm{C}$ infection. Serum level of carcinoembryonic antigen (CEA) and cancer antigen (CA) 19-9 was also included. Data regarding treatment details were collected including type of surgery and receipt of adjuvant chemotherapy and radiotherapy. The type of surgery was classified as wedge liver resection, minor resection (removal of $\leq 2$ Couinaud segment), and major resection (removal of $\geq 3$ Couinaud segment) $\left[{ }^{17}\right]$. Resection margin status was recorded and classified as microscopically negative (R0) and microscopically positive (R1). Tumor-specific characteristics including tumor size, number, grade, number of lymph nodes harvested, number of metastatic nodes, presence of vascular invasion (macroscopic and microscopic), perineural invasion, biliary invasion, and direct invasion of contiguous organs were obtained. Lymphadenectomy was performed such that the regional lymph nodes including the nodes in the hepatoduodenal ligament (station 12), the nodes along the common hepatic artery (station 8), and the nodes on the posterior surface of the head of the pancreas (station 13) were harvested, in addition, for left side ICC, also the nodes along the trunk of left gastric artery (station 7). Data on tumor stage 
were also collected according to both the seventh and the eighth edition AJCC staging system $\left[{ }^{1,16}\right]$. Nodal status was assessed preoperatively by endoscopic ultrasound echography (EUS), computer tomography (CT) scan, magnetic resonance imaging (MRI), and positron emission tomography (PET) scan; nodes were classified on preoperative imaging as positive, negative, or suspicious. Date of last follow-up and vital status were collected on all patients.

\section{Statistical Analysis}

Continuous variables were reported as medians with interquartile ranges (IQRs), while categorical variables were reported as whole numbers and percentages. The endpoint for the survival analysis was overall survival (OS). OS was defined as the time interval between the date of surgery and the date of death. Time was censored at the date of the last followup assessment for patients who were still alive at the time of analysis. Survival curves were estimated using the KaplanMeier method, and differences between the curves were compared using the log-rank test. Univariate Cox proportional hazard models were used to evaluate associations between variables and OS. The coefficients from the Cox models were reported as hazard ratios (HRs) with corresponding 95\% confidence intervals (CIs). A Bayesian model was developed to analyze the prognosis of patients with or without metastatic nodes (N1 versus N0) based on the number of harvested nodes $\left[{ }^{18}\right]$. The results of the Bayesian model were presented as 5year OS and HR with associated 95\% credible intervals $(\mathrm{Cr}$ Is). All analyses were performed using the statistical software programs STATA (v. 12.0, StataCorp, College Station, TX), OpenBugs (v.2011), and R CRAN (v. 3.2.2, 2015) with the packages "survival," "Hmisc," and "R2OpenBUGS." A $p$ value $<0.10$ was considered statistically significant.

\section{Results}

\section{Baseline Characteristics of the Study Group}

Among 1154 patients who underwent hepatectomy for ICC, most patients were male $(n=638,55.3 \%)$ and younger than 65 years $(n=712,61.7 \%$; Table 1$)$. Based on the ASA physical status classification, 634 (54.9\%) patients were ASA $\leq 2$ and $520(45.1 \%)$ patients were ASA 3 or 4 . Preoperatively, a minority of patients $(n=84,7.3 \%)$ underwent neoadjuvant chemotherapy. About two thirds of patients $(n=708$, $61.4 \%)$ underwent a major hepatectomy, while 157 (13.6\%) underwent a wedge resection and $289(25.0 \%)$ a minor hepatectomy. A resection with negative margins (R0) was performed in the majority of patients $(n=992,87.2 \%)$, while $146(12.8 \%)$ patients had R1 resections. Mass forming
Table 1 Baseline characteristics of the study group $(n=1154)$

\begin{tabular}{|c|c|}
\hline Variables & $N(\%)$ \\
\hline \multicolumn{2}{|l|}{ Gender } \\
\hline Female & $516(44.7 \%)$ \\
\hline Male & $638(55.3 \%)$ \\
\hline \multicolumn{2}{|l|}{ Age } \\
\hline$\leq 65$ years & $712(61.7 \%)$ \\
\hline$>65$ years & $442(38.3 \%)$ \\
\hline \multicolumn{2}{|l|}{ ASA score } \\
\hline$\leq 2$ & $634(54.9 \%)$ \\
\hline$>2$ & $520(45.1 \%)$ \\
\hline \multicolumn{2}{|l|}{ Underlying liver disease } \\
\hline Cirrhosis & $118(10.2 \%)$ \\
\hline Chronic HBV infection & $129(11.2 \%)$ \\
\hline Chronic HCV infection & $15(1.3 \%)$ \\
\hline None & $892(77.3 \%)$ \\
\hline \multicolumn{2}{|l|}{ Neoadjuvant chemotherapy } \\
\hline No & $1065(92.7 \%)$ \\
\hline Yes & $84(7.3 \%)$ \\
\hline \multicolumn{2}{|l|}{ Morphological type } \\
\hline Mass forming (MF) & $941(87.0 \%)$ \\
\hline Periductal infiltrating (PI) & $54(4.9 \%)$ \\
\hline $\mathrm{MF}+\mathrm{PI}$ & $88(8.1 \%)$ \\
\hline NA & 71 \\
\hline Ca 19-9, median (IQR) & $49 \mathrm{U} / \mathrm{mL}(16.9-204.0)$ \\
\hline CEA, median (IQR) & $2.4 \mathrm{ng} / \mathrm{mL}(1.4-4.3)$ \\
\hline \multicolumn{2}{|l|}{ Type of surgery } \\
\hline Wedge resection & $157(13.6 \%)$ \\
\hline Minor hepatectomy & $289(25.0 \%)$ \\
\hline Major hepatectomy & $708(61.4 \%)$ \\
\hline \multicolumn{2}{|l|}{ Margins } \\
\hline Negative & $992(87.2 \%)$ \\
\hline Positive & $146(12.8 \%)$ \\
\hline NA & 16 \\
\hline \multicolumn{2}{|l|}{ Liver capsule involvement } \\
\hline No & $945(81.9 \%)$ \\
\hline Yes & $209(18.1 \%)$ \\
\hline \multicolumn{2}{|l|}{ Tumor size } \\
\hline$\leq 5 \mathrm{~cm}$ & $451(39.1 \%)$ \\
\hline$>5 \mathrm{~cm}$ & $703(60.9 \%)$ \\
\hline \multicolumn{2}{|l|}{ Lesion } \\
\hline Unifocal & $941(81.5 \%)$ \\
\hline Multifocal & $213(18.5 \%)$ \\
\hline \multicolumn{2}{|l|}{ Grade } \\
\hline Well-moderate & $884(82.5 \%)$ \\
\hline Poor-undifferentiated & $188(17.5 \%)$ \\
\hline NA & 82 \\
\hline \multicolumn{2}{|l|}{ Major vascular invasion } \\
\hline Not present & $998(86.5 \%)$ \\
\hline Present & $156(13.5 \%)$ \\
\hline \multicolumn{2}{|l|}{ Lymph-vascular invasion } \\
\hline Not present & $771(69.2 \%)$ \\
\hline Present & $356(30.8 \%)$ \\
\hline NA & 27 \\
\hline \multicolumn{2}{|l|}{ Perineural invasion } \\
\hline Not present & $805(78.9 \%)$ \\
\hline Present & $215(21.1 \%)$ \\
\hline NA & 134 \\
\hline \multicolumn{2}{|c|}{ Preoperative imaging for nodal staging } \\
\hline Echography & $19(2.2 \%)$ \\
\hline $\mathrm{CT}$ & $436(51.3 \%)$ \\
\hline MRI & $319(37.5 \%)$ \\
\hline $\mathrm{PET} / \mathrm{CT}$ & $76(9.0 \%)$ \\
\hline NA & 304 \\
\hline Radiological nodal status & \\
\hline Negative & $608(52.7 \%)$ \\
\hline
\end{tabular}


Table 1 (continued)

\begin{tabular}{ll}
\hline Variables & $N(\%)$ \\
\hline Suspicious & $118(10.2 \%)$ \\
Positive & $59(5.1 \%)$ \\
Not reported & $369(32.0 \%)$ \\
Pathological nodal status & $315(27.3 \%)$ \\
Negative & $200(17.3 \%)$ \\
Metastatic & $639(55.4 \%)$ \\
Not harvested & $4(2-8)$ \\
Lymph nodes harvested ${ }^{\mathrm{a}}$, median (IQR) & \\
Metastatic lymph nodes & \\
1 & $110(55.0 \%)$ \\
$2-5$ & $65(32.5 \%)$ \\
$\geq 6$ & $25(12.5 \%)$ \\
AJCC eighth edition N status & \\
N0 & $117(10.1 \%)$ \\
N1 & $200(17.3 \%)$ \\
NX & $837(72.6 \%)$ \\
AJCC seventh edition T status & \\
T1 & $487(42.2 \%)$ \\
T2a & $207(17.9 \%)$ \\
T2b & $123(10.7 \%)$ \\
T3 & $195(16.9 \%)$ \\
T4 & $142(12.3 \%)$ \\
AJCC eighth edition T status & \\
T1a & $249(21.6 \%)$ \\
T1b & $270(23.4 \%)$ \\
T2 & $402(34.8 \%)$ \\
T3 & $167(14.5 \%)$ \\
T4 & $66(5.7 \%)$ \\
\hline
\end{tabular}

$N A$ not available, $I Q R$ interquartile range

${ }^{a}$ Of 515 patients who underwent lymphadenectomy

${ }^{\mathrm{b}}$ Of 200 patients with metastatic nodes;

( $n=941,87.0 \%$ ) was the most common morphological subtype of ICC. Overall, 941 (81.5\%) patients had a single tumor, while $213(18.5 \%)$ patients had multifocal disease. According to the AJCC seventh edition T staging system, 487 (42.2\%) patients were classified as stage T1, $207(17.9 \%)$ as stage T2a, $123(10.7 \%)$ as stage T2b, $195(19.9 \%)$ as stage T3, and 142 $(12.3 \%)$ as stage T4. Using the AJCC eighth edition T staging system, $249(21.6 \%)$ patients were classified as stage T1a, 270 $(23.4 \%)$ as T1b, $402(34.8 \%)$ as T2, $167(14.5 \%)$ as T3, and $66(5.7 \%)$ as T4.

\section{Radiological Nodal Status}

A total of $785(68.0 \%)$ patients had data on preoperative radiological nodal staging. EUS, CT, MRI, and PET were used to preoperatively assess nodal status in 19 (2.4\% of 785$), 383$ (48.9\% of 785 ), 307 (39.1\% of 785), and 76 (9.7\% of 785 ) patients, respectively. Among radiological-staged patients, nodal status was negative (R-NLN) in 608 (77.5\% of 785) patients, suspicious (R-SLN) in 118 (15.0\% of 785), and metastatic (R-MLN) in 59 (7.5\% of 785) patients. Patients with RNLN had a 5-year OS of 49.7\% (IQR, 44.3-54.8) compared with a 5 -year OS of $30.1 \%$ (IQR, 18.7-42.4) for patients with
R-SLN and 25.8\% (IQR, 12.5-41.4) for patients with R-MLN $(p<0.001$; Table 2, Fig. S1). Compared with patients with RNLN, patients with R-SLN (HR 1.55, 95\% CI, 1.15-2.06; $p=0.003$ ) and R-MLN (HR 1.82, 95\% CI, 1.27-2.62; $p=0.001)$ were at higher risk of death (Table 2).

Among 317 (27.5\%) patients who had data on both radiological and pathological nodal evaluation, the incidence of NLN was $66.5 \%(n=127)$ among patients initially deemed R-NLN compared with $42.5 \%(n=34)$ among patients who were preoperatively staged R-SLN; in contrast, the incidence of NLN was only $34.8 \%(n=16)$ among patients deemed preoperatively to be R-MLN. The incidence of MLN increased from $33.5 \%(n=64)$ among patients who were R-NLN to $57.5 \%$ $(n=46)$ and $65.2 \%(n=30)$ among patients who were R-SLN or R-MLN, respectively ( $p<0.001$; Table 3 ). Radiological nodal status was associated with advanced disease (T3/T4 AJCC seventh ed. and eighth ed. stages; both $p \leq 0.003$; Table 3). Of note, the area under the receiver operating characteristic (ROC) curve comparing nodes deemed preoperatively as positive or suspicious versus documented metastatic nodal disease on final pathology was 0.63 .

\section{Nodal Status}

At the time of hepatectomy, nodes were harvested in 515 (44.6\%) patients, while $639(55.4 \%)$ patients did not undergo lymphadenectomy. When lymphadenectomy was performed, the median number of harvested lymph node (HLN) was 4 (IQR, 2-8). Overall, 200 (17.3\%) patients had metastatic lymph nodes (MLNs), and 315 (27.3\%) patients had no evidence of lymph node metastasis (NLN). Among the 200 (17.3\%) patients with MLN, 110 (55.0\% of MLN group) patients had 1 MLN, 65 (32.5\% of MLN group) 2-5 MLNs, and 25 (12.5\% of MLN group) $\geq 6$ MLNs. Among the $315(27.3 \%)$ patients with NLN, 67 (21.3\% of NLN group) patients had 1 HLN, 131 (41.6\% of NLN group) 2-5 HLNs, and 117 (37.1\% of NLN group) $\geq 6$ HLNs. The 5 -year OS of patients with NLN was $44.4 \%$ (IQR, 36.9-51.6) versus $15.2 \%$ (IQR, 8.723.4) for patients with MLN (HR 2.42, 95\% CI 1.88-3.13; $p<0.001$; Fig. 1).

To verify the prognostic role of six HLNs as the minimum number recommended by the AJCC eighth edition staging system for an adequate nodal staging, MLN and NLN patients were dichotomized in two groups with a cutoff of six HLNs. Among the $200(17.3 \%)$ patients with MLN, 100 (50.0\% of MLN group) patients had $\geq 6$ HLNs with a 5 -year OS of $17.9 \%$ (IQR, 8.6-29.9) versus a 5-year OS of $12.5 \%$ among the 100 (50.0\% of MLN group) patients who had $<6 \mathrm{HLNs}$ (IQR, 4.7-24.2; $p=0.71$; Fig. S2). Patients with MLN had a similar risk of death when stratified by $<6$ versus $\geq 6$ HLNs (HR 1.07, 95\% CI 0.75-1.52, $p=0.72$ ). Among the 315 (27.3\%) patients with NLN, 117 (37.1\% of NLN group) patients had $\geq 6$ HLNs with a 5 -year OS of $54.9 \%$ (IQR, 41.6- 
Table 2 Comparison of nodal status - Kaplan-Meier analysis and risk of death

\begin{tabular}{|c|c|c|c|c|c|c|c|}
\hline & $N=1154$ & 5-Year OS & $95 \% \mathrm{CI}$ & $p$ value & $\mathrm{HR}$ & $95 \% \mathrm{CI}$ & $p$ value \\
\hline Radiological nodal status & & & & $<0.001$ & & & \\
\hline Negative & $608(52.7 \%)$ & $49.7 \%$ & $44.3-54.8$ & & - & - & - \\
\hline Suspicious & $118(10.2 \%)$ & $30.1 \%$ & $18.7-42.4$ & & 1.55 & $1.15-2.06$ & 0.003 \\
\hline Positive & $59(5.1 \%)$ & $25.8 \%$ & $12.5-41.4$ & & 1.82 & $1.27-2.62$ & 0.001 \\
\hline Not reported & $369(32.0 \%)$ & $30.7 \%$ & $25.3-36.2$ & & 1.57 & $1.30-1.90$ & $<0.001$ \\
\hline Pathological nodal status & & & & $<0.001$ & & & \\
\hline Negative & $315(27.3 \%)$ & $44.4 \%$ & $36.9-51.6$ & & - & - & - \\
\hline Metastatic & $200(17.3 \%)$ & $15.2 \%$ & $8.7-23.4$ & & 2.42 & $1.88-3.13$ & $<0.001$ \\
\hline Not harvested & $639(55.4 \%)$ & $44.0 \%$ & $39.2-48.7$ & & 1.18 & $0.96-1.47$ & 0.11 \\
\hline AJCC eighth edition $\mathrm{N}$ stage & & & & $<0.001$ & & & \\
\hline No & $117(10.1 \%)$ & $54.9 \%$ & $41.6-66.3$ & & - & - & - \\
\hline $\mathrm{N} 1$ & $200(17.3 \%)$ & $15.2 \%$ & $8.7-23.4$ & & 3.03 & $2.08-4.42$ & $<0.001$ \\
\hline NX & $837(72.6 \%)$ & $42.9 \%$ & $38.6-47.1$ & & 1.46 & $1.04-2.07$ & 0.031 \\
\hline Negative nodal status & & & & 0.098 & & & \\
\hline NLN with $\geq 6$ HLNs & $117(37.1 \%)$ & $54.9 \%$ & $41.6-66.3$ & & - & - & - \\
\hline NLN with $<6$ HLNs & $198(62.9 \%)$ & $39.4 \%$ & $30.6-48.1$ & & 1.39 & $0.94-2.07$ & 0.098 \\
\hline Metastatic nodal status & & & & 0.72 & & & \\
\hline MLN with $\geq 6$ HLNs & $100(50.0 \%)$ & $17.9 \%$ & $8.6-29.9$ & & - & - & - \\
\hline MLN with $<6$ HLNs & $100(50.0 \%)$ & $12.5 \%$ & $4.7-24.2$ & & 1.07 & $0.75-1.52$ & 0.72 \\
\hline Radiological nodal status in NHN & & & & $<0.001$ & & & \\
\hline Negative & $417(65.3 \%)$ & $52.3 \%$ & $46.1-58.2$ & & - & - & - \\
\hline Suspicious & $38(5.9 \%)$ & $24.8 \%$ & $9.3-44.2$ & & 1.55 & $1.15-2.07$ & 0.003 \\
\hline Positive & $13(2.0 \%)$ & $20.1 \%$ & $3.3-47.1$ & & 1.82 & $1.27-2.62$ & 0.001 \\
\hline Not reported & $171(26.8 \%)$ & $32.1 \%$ & $24.3-40.0$ & & 1.58 & $1.31-1.90$ & $<0.001$ \\
\hline
\end{tabular}

$O S$ overall survival, $C I$ confidence interval, $H L N$ harvested lymph node, $M L N$ metastatic lymph node

66.3) compared with a 5-year OS of 39.4\% (IQR, 30.6-48.1; $p=0.098$; Fig. S3) among the 198 (62.9\% of NLN group) patients who had $<6$ HLNs. Interestingly, patients with NLN who had $<6$ nodes harvested tended to have an increased risk of death compared with patients who had $\geq 6$ HLNs (HR 1.39, 95\% CI 0.94-2.07, $p=0.098$ ).

\section{Patients Without Nodes Harvested}

A total of 639 (55.4\%) patients did not undergo lymphadenectomy $(\mathrm{Nx})$; Nx patients had a 5-year OS of 44.0\% (IQR, 39.248.7) (HR 1.18, 95\% CI 0.96-1.47; $p=0.11$ ) (Table 2). When pathological $\mathrm{Nx}$ patients were stratified according to
Table 3 Association between radiological nodal status, pathological nodal status, AJCC seventh ed., and AJCC eighth ed. T stages

\begin{tabular}{|c|c|c|c|c|}
\hline & \multicolumn{3}{|c|}{ Radiological modal status } & \multirow[t]{2}{*}{$p$ value } \\
\hline & Negative & Suspicious & Positive & \\
\hline Pathological nodal status ${ }^{\mathrm{a}}$ & & & & $<0.001$ \\
\hline Negative & $127(66.5 \%)$ & $34(42.5 \%)$ & $16(34.8 \%)$ & \\
\hline Positive & $64(33.5 \%)$ & $46(57.5 \%)$ & $30(65.2 \%)$ & \\
\hline AJCC seventh edition T stages ${ }^{b}$ & & & & $<0.001$ \\
\hline $\mathrm{T} 1 / \mathrm{T} 2 \mathrm{a} / \mathrm{T} 2 \mathrm{~b}$ & $471(77.5 \%)$ & $74(62.7 \%)$ & $31(52.5 \%)$ & \\
\hline $\mathrm{T} 3 / \mathrm{T} 4$ & $137(22.5 \%)$ & $44(37.3 \%)$ & $28(47.5 \%)$ & \\
\hline AJCC eighth edition T stages ${ }^{\mathrm{b}}$ & & & & 0.003 \\
\hline $\mathrm{T} 1 \mathrm{a} / \mathrm{T} 1 \mathrm{~b} / \mathrm{T} 2$ & $406(66.8 \%)$ & $70(59.3 \%)$ & $27(45.8 \%)$ & \\
\hline $\mathrm{T} 3 / \mathrm{T} 4$ & $202(33.2 \%)$ & $48(40.7 \%)$ & $32(54.2 \%)$ & \\
\hline
\end{tabular}

${ }^{\mathrm{a}} n=317$ patients

${ }^{\mathrm{b}} n=785$ patients 


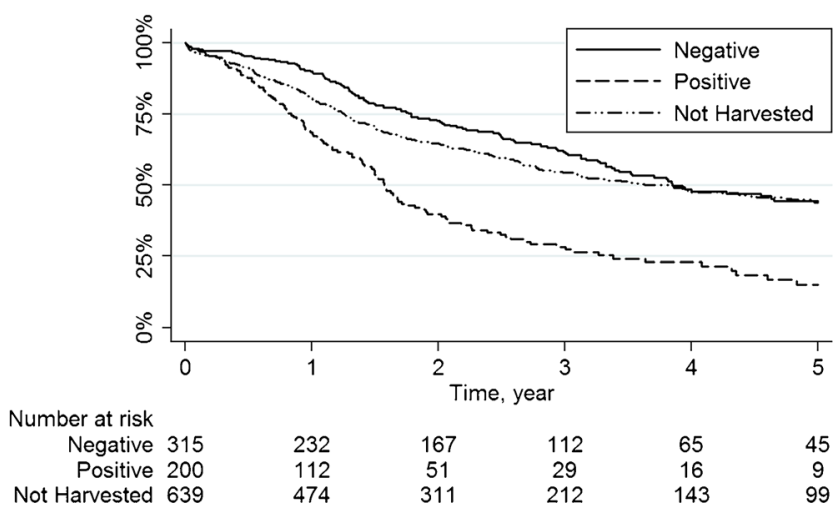

Fig. 1 Kaplan-Meier overall survival curves stratified by pathological nodal status

preoperative radiological nodal status, $417(65.3 \%)$ were in the R-NLN group, 38 (5.9\%) in the R-SLN, and 13 (2.0\%) in the R-MLN group; 171 (26.8\%) did not have any information on radiological node status (R-Nx). Among pathological Nx patients, 5-year OS among patients with R-NLN was $52.3 \%$ (IQR, 46.1-58.2) versus $24.8 \%$ (IQR, 9.3-44.2) for R-SLN, 20.1\% (IQR, 3.3-47.1) for R-MLN and 32.1\% (IQR, 24.3-40.0) for R-Nx (Table 2). Compared with patients who had R-NLN, patients with R-SLN (HR 1.55, 95\% CI 1.15-2.07), R-MLN (HR 1.82, 95\% CI 1.27-2.62), and RNx (HR 1.58, 95\% CI 1.31-1.90) had an increased hazard of death (all $p \leq 0.003$; Table 2).

\section{AJCC Eighth Nodal Staging}

Among the 317 (27.5\%) patients with MLN or NLN with $\geq 6$ HLNs - the cutoff recommended by the AJCC eighth edition staging system-117 (36.9\% of 317 ) patients were defined as N0 and $200(63.1 \%$ of 317$)$ as N1. The 5 -year OS of N0 patients was $54.9 \%$ (IQR, 41.6-66.3; Table 2) versus $15.2 \%$ (IQR, 8.7-23.4) for N1 patients ( $p<0.001$; Fig. 2). In turn, N1 patients had an increased hazard of death compared with $\mathrm{N} 0$ patients (HR 3.03, 95\% CI 2.08-4.42; $p<0.001$ ).

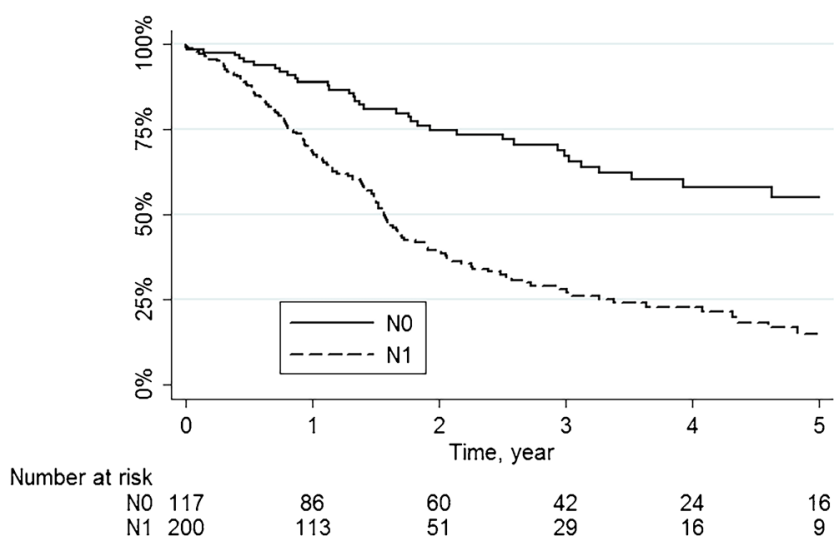

Fig. 2 Kaplan-Meier overall survival curves stratified by AJCC eighth edition nodal stages
To further investigate the effect of the number of HLN on the prognosis of N0 and N1 patients, a Bayesian Weibull model was developed. N1 patients had an increased risk of death (HR 2.42, 95\% Cr Int, 1.69-3.38) compared with N0 patients who had one HLN. Of note, when the number of HLN was increased to $3,6,8$, and 10 , the risk of death among $\mathrm{N} 1$ patients versus N0 patients increased incrementally to 2.61, 2.89, 3.11, and 3.34, respectively. Specifically, 5-year OS among N0 patients increased with higher numbers of HLN from $38.3 \%$ with 1 HLN to $42.7,49.2,53.4$, and $57.3 \%$ with $3,6,8$, and 10 HLNs, respectively. Conversely, 5 -year OS among N1 patients did not varying considerably when HLN increased, ranging from $9.7 \%$ with 1 HLN to only $15.5 \%$ with 10 HLNs (Table S1).

\section{Discussion}

Several studies have investigated a variety of clinicopathological factors and long-term survival, identifying tumor size, tumor number, vascular invasion, and lymph node metastasis as independent predictors of OS and recurrence-free survival (RFS) among patients undergoing surgery for ICC [2, 15, 19, $\left.{ }^{20}\right]$. In particular, the role of lymphadenectomy for ICC has been extensively debated $\left[{ }^{2,8-10}\right]$. In a recent meta-analysis that evaluated the management of lymph node basin during liver resection for ICC, the authors suggested that surgeons should strongly consider lymph node dissection at the time of surgery although there was insufficient data to support a strong recommendation for routine lymphadenectomy $\left[{ }^{21}\right]$. The newly released eighth edition AJCC staging manual advocates, however, for recovery of at least six lymph nodes during the time of surgery for ICC $\left[{ }^{16}\right]$. Given the large variability in lymphadenectomy among Eastern and Western centers, little data exist regarding the optimal number of lymph nodes to harvest at the time of surgery for ICC. In addition, no study has explicitly sought to evaluate the prognostic relevance of preoperative radiological versus pathological lymph node status. While several studies have evaluated the sensitivity, specificity, and predictive value of ultrasound, CT, MRI, and PET $\left[{ }^{22-24}\right]$, no study had assessed the prognostic ability of preoperatively determine $\mathrm{N}$ status and long-term outcomes following resection of ICC. The current study was important, therefore, as we determined the prognostic impact of preoperative radiologic nodal status. In addition, we assessed the eighth AJCC edition's recommendation for a minimum recovery of six lymph nodes and its association with long-term outcome among a large multiinstitutional cohort of patients with ICC.

In addition to pathological staging, preoperative radiological lymph node status, as assessed by EUS, CT, MRI, or PET, was correlated with long-term prognosis among patients undergoing resection of ICC (Table 2). Specifically, patients who 
had metastatic lymph node disease on preoperative imaging had a 5 -year OS of only $25.8 \%$, which was almost one half the 5 -year OS (49.7\%) among patients who had no lymph node disease suspected on preoperative imaging. Interestingly, patients who had nodes deemed as suspicious on preoperative imaging had a comparable 5-year outcome (30.1\%) as patients who had metastatic nodes. In fact, patients who had nodes deemed as positive or suspicious on preoperative imaging had an 82 and 55\% increased hazard of death long-term compared with patients who had no nodal disease.

Perhaps not surprisingly, pathological nodal status also was associated with prognosis and, in fact, was much more strongly correlated with long-term outcome than preoperative lymph node assessment. Specifically, patients with pathologic N1 disease according to the AJCC eighth edition staging system had almost a 2.5 -fold increased risk of death at 5 years compared with N0 patients. The superiority of pathological versus radiological nodal status to predict long-term survival might be expected. While preoperative imaging can often accurately predict "true" nodal status, some inaccuracy and lack of correlation of preoperative imaging with final pathology can occur. Specifically, in the current study, the area under the ROC curve comparing nodes that were "positive" or "suspicious" on preoperative imaging with true nodal disease on final pathology was 0.63 . These data suggest that the correlation of preoperative imaging to detect nodal disease was good to fair. Moreover, radiological node status was also correlated with both AJCC seventh and eighth editions' T stages, as patients with advanced $\mathrm{T}$ stage disease were more likely to have positive or suspicious nodes on the preoperative imaging (Table 3).

Recently, the AJCC eighth edition proposed a cutoff of six lymph nodes to $\mathrm{N}$ stage patients adequately. The impact of number of nodes examined has not been examined among patients undergoing resection of ICC. As such, we investigated the effect of a cutoff of six nodes on long-term outcome. Of note, OS among patients with N0 disease who had $\geq 6$ HLNs was $54.9 \%$, which was markedly better than the $15.2 \%$ survival noted among patient with $\mathrm{N} 1$ disease. Perhaps of more interest, when assessed using a Bayesian Weibull model, the 5 -year OS of N0 patients improved with increasing numbers of HLN, while the 5-year OS of N1 patients did not change with a higher number of HLN (Table S1). In addition, while the 5 -year OS of patients with N1 disease who had $\geq 6$ HLNs was no different than patients who had $<6 \mathrm{HLNs}$, the 5 -year OS of patients with N0 disease with $<6$ HLNs was somewhat worse versus N0 patients who had a more thorough lymph node harvest of $\geq 6$ nodes.

The study had several limitations that should be considered when interpreting the results. While one strength of the current report was that it involved multiple centers, the multiinstitutional nature likely led to different radiological imaging techniques. In turn, the sensitivity and specificity of these different techniques in staging the nodal basin may vary $\left.{ }^{23-25}\right]$. The multicenter nature of the study also did not allow for standardization of operative or perioperative approach, especially in terms of performance and extent of lymphadenectomy. Finally, the long duration and the multiinstitutional nature of the study likely caused some heterogeneity in ICC treatment approach. However, given the rarity of ICC, obtaining data from multiple centers increased the sample size and made the data more generalizable.

\section{Conclusion}

In conclusion, while pathological nodal status was strongly associated with long-term outcome, only one fourth of patients undergoing liver resection for ICC had adequate nodal staging according to the newly released AJCC eighth edition staging system. Moreover, our results suggested that radiological lymph node staging could be inaccurate in up to $40 \%$ of patients and should not be considered a valid alternative to lymphadenectomy. Furthermore, the best ability to discriminate between patients with favorable prognosis and patients with poor prognosis based on the lymph node status was reached when $\geq 6$ lymph nodes were harvested. In other words, the quality of the lymph node staging in terms of hazard of death of metastatic patients compared with non-metastatic patients increased from 1.6 to 1.8 with radiological assessment to 2.4 with pathology assessment; of note, the hazard of death increases to 3-fold when the AJCC eighth ed. staging system recommendations were fulfilled. While the six HLN cutoff value was associated with prognosis among patients staged as N0, the number of HLN was not associated with longterm survival among patients with $\mathrm{N} 1$ disease. These data serve to emphasize the important prognostic role of pathological staging of nodal disease among patients undergoing resection of ICC.

\section{Compliance with Ethical Standards}

Conflict of Interest The authors declare that they have no conflict of interest.

\section{References}

1. Edge SB BD, Compton CC, Fritz AG, Greene FL, Trotti A. AJCC Cancer Staging Manual (7th edn). 2010.

2. de Jong MC, Nathan H, Sotiropoulos GC, et al. Intrahepatic cholangiocarcinoma: an international multi-institutional analysis of prognostic factors and lymph node assessment. Journal of clinical oncology : official journal of the American Society of Clinical Oncology. 2011;29(23):3140-3145.

3. Farges O, Fuks D, Le Treut YP, et al. AJCC 7th edition of TNM staging accurately discriminates outcomes of patients with resectable intrahepatic cholangiocarcinoma: By the AFC-IHCC-2009 study group. Cancer. 2011;117(10):2170-2177. 
4. Igami T, Ebata T, Yokoyama Y, Sugawara G, Takahashi Y, Nagino M. Staging of peripheral-type intrahepatic cholangiocarcinoma: appraisal of the new TNM classification and its modifications. World journal of surgery. 2011;35(11):2501-2509.

5. Ali SM, Clark CJ, Mounajjed T, et al. Model to predict survival after surgical resection of intrahepatic cholangiocarcinoma: the Mayo Clinic experience. HPB : the official journal of the International Hepato Pancreato Biliary Association. 2015;17(3): 244-250.

6. Jiang W, Zeng ZC, Tang ZY, et al. A prognostic scoring system based on clinical features of intrahepatic cholangiocarcinoma: the Fudan score. Annals of oncology : official journal of the European Society for Medical Oncology / ESMO. 2011;22(7):1644-1652.

7. Hyder O, Marques H, Pulitano C, et al. A Nomogram to Predict Long-term Survival After Resection for Intrahepatic Cholangiocarcinoma: An Eastern and Western Experience. JAMA surgery. Mar 52014.

8. Clark CJ, Wood-Wentz CM, Reid-Lombardo KM, Kendrick ML, Huebner M, Que FG. Lymphadenectomy in the staging and treatment of intrahepatic cholangiocarcinoma: a population-based study using the National Cancer Institute SEER database. HPB : the official journal of the International Hepato Pancreato Biliary Association. 2011;13(9):612-620.

9. Mavros MN, Economopoulos KP, Alexiou VG, Pawlik TM. Treatment and Prognosis for Patients With Intrahepatic Cholangiocarcinoma: Systematic Review and Meta-analysis. JAMA surgery. Apr 92014.

10. Kim Y, Spolverato G, Amini N, et al. Surgical Management of Intrahepatic Cholangiocarcinoma: Defining an Optimal Prognostic Lymph Node Stratification Schema. Annals of surgical oncology. 2015;22(8):2772-2778.

11. Vitale A, Moustafa M, Spolverato G, Gani F, Cillo U, Pawlik TM. Defining the possible therapeutic benefit of lymphadenectomy among patients undergoing hepatic resection for intrahepatic cholangiocarcinoma. Journal of surgical oncology. 2016;113(6):685-691.

12. Nuzzo G, Giuliante F, Ardito F, et al. Improvement in perioperative and long-term outcome after surgical treatment of hilar cholangiocarcinoma: results of an Italian multicenter analysis of 440 patients. Archives of surgery. 2012;147(1):26-34.

13. Shimada M, Yamashita Y, Aishima S, Shirabe K, Takenaka K, Sugimachi K. Value of lymph node dissection during resection of intrahepatic cholangiocarcinoma. The British journal of surgery. 2001;88(11):1463-1466.

14. Bagante F, Gani F, Spolverato G, et al. Intrahepatic Cholangiocarcinoma: Prognosis of Patients Who Did Not Undergo Lymphadenectomy.
Journal of the American College of Surgeons. 2015;221(6):1031-1040 e1034.

15. Bridgewater J, Galle PR, Khan SA, et al. Guidelines for the diagnosis and management of intrahepatic cholangiocarcinoma. Journal of hepatology. 2014;60(6):1268-1289.

16. Amin MB EiC, American Joint Committee on Cancer. Springer. 2017.

17. Couinaud C. Liver anatomy: portal (and suprahepatic) or biliary segmentation. Digestive surgery. 1999;16(6):459-467.

18. Bagante F, Tran T, Spolverato G, et al. Perihilar Cholangiocarcinoma: Number of Nodes Examined and Optimal Lymph Node Prognostic Scheme. Journal of the American College of Surgeons. 2016;222(5): 750-759 e 752.

19. Hyder O, Marques H, Pulitano C, et al. A nomogram to predict long-term survival after resection for intrahepatic cholangiocarcinoma: an Eastern and Western experience. JAMA surgery. 2014;149(5):432-438.

20. Doussot A, Gonen M, Wiggers JK, et al. Recurrence Patterns and Disease-Free Survival after Resection of Intrahepatic Cholangiocarcinoma: Preoperative and Postoperative Prognostic Models. Journal of the American College of Surgeons. 2016;223(3):493-505 e492.

21. Amini N, Ejaz A, Spolverato G, Maithel SK, Kim Y, Pawlik TM. Management of lymph nodes during resection of hepatocellular carcinoma and intrahepatic cholangiocarcinoma: a systematic review. Journal of gastrointestinal surgery : official journal of the Society for Surgery of the Alimentary Tract. 2014;18(12):2136 2148.

22. Holzapfel K, Gaa J, Schubert EC, et al. Value of diffusion-weighted MR imaging in the diagnosis of lymph node metastases in patients with cholangiocarcinoma. Abdominal radiology. 2016;41(10): 1937-1941.

23. Hanninen EL, Pech M, Jonas S, et al. Magnetic resonance imaging including magnetic resonance cholangiopancreatography for tumor localization and therapy planning in malignant hilar obstructions. Acta radiologica. 2005;46(5):462-470.

24. Kluge R, Schmidt F, Caca K, et al. Positron emission tomography with [(18)F]fluoro-2-deoxy-D-glucose for diagnosis and staging of bile duct cancer. Hepatology. 2001;33(5):1029-1035.

25. Roche CJ, Hughes ML, Garvey CJ, et al. CT and pathologic assessment of prospective nodal staging in patients with ductal adenocarcinoma of the head of the pancreas. AJR. American journal of roentgenology. 2003;180(2):475-480. 